\title{
Search for Squarks in $R$-parity Violating Supersymmetry in ep Collisions at HERA
}

\section{Gerhard Brandt ${ }^{* \dagger}$}

University of Oxford - Denys Wilkinson Building, Keble Road, Oxford OXI 3RH

E-mail: grbrandt@cern.ch

\begin{abstract}
This talk presents a search for squarks in $R$-parity violating supersymmetry in $e^{ \pm} p$ collisions at HERA using the H1 detector. The resonant production of squarks via a Yukawa coupling $\lambda^{\prime}$ is considered, taking into account direct and indirect $R$-parity violating decay modes. Final states with jets and leptons are investigated. No evidence for squark production is found and mass dependent limits on $\lambda^{\prime}$ are obtained in the framework of the Minimal Supersymmetric Standard Model and in the Minimal Supergravity Model. In the considered part of the parameter space, for a Yukawa coupling of electromagnetic strength $\lambda^{\prime}=0.3$, squarks of all flavours are excluded up to masses of $275 \mathrm{GeV}$ at $95 \%$ confidence level, with down-type squarks further excluded up to masses of $290 \mathrm{GeV}$.
\end{abstract}

36th International Conference on High Energy Physics

4-11 July 2012

Melbourne, Australia

\footnotetext{
* Speaker.

†n behalf of the $\mathrm{H} 1$ collaboration.
} 
This talk presents a search for squark-production at the world's only ep collider HERA [1]. In supersymmetric (SUSY) models with $R$-parity violation $\left(R_{p}^{\prime}\right)$, squarks can couple to electrons and quarks via Yukawa couplings $\lambda^{\prime}$. At HERA, squarks with masses up to the electron-proton centre-of-mass energy, $\sqrt{s}=319 \mathrm{GeV}$, could be produced resonantly via the fusion of the incoming $27.6 \mathrm{GeV}$ electron and a quark from the incoming $920 \mathrm{GeV}$ proton. The data used in this analysis correspond to an integrated luminosity of $255 \mathrm{pb}^{-1}$ for $e^{+} p$ collisions and $183 \mathrm{pb}^{-1}$ for $e^{-} p$ collisions which represents the full data sample collected at $\sqrt{s}=319 \mathrm{GeV}$.

In the considered phenomenological minimal supersymmetric Standard Model (MSSM) squarks are produced via the $R_{p}$ couplings $\lambda_{i j k}^{\prime}$ where $i, j, k=1,2,3$ are generation indices. Only the couplings $\lambda_{1 j 1}^{\prime}$ and $\lambda_{11 k}^{\prime}$ are relevant at HERA. Squarks decay either directly via the same couplings, or via cascade decays via $R_{p}$ conserving decays with subsequent $R$-parity violating $\chi^{0} / \chi^{ \pm}$decay.

A total of 8 (9) exclusive final state topologies are investigated in the $e^{+} p\left(e^{-} p\right)$ collision data, classified by the presence of identified electrons (e), muons $(\mu)$, neutrinos $(v)$, a jet $(q)$ or multiple jets $(M J)$. Direct squark decays lead to $e q$ and $v q$ final states, with irreducible background from neutral and charged currents in deep-inelastic scattering. Cascade squark decays lead to $e(e / \mu) M J$ and $v(e / \mu) M J$ final states. In each topology, in addition to the required presence of the final state objects, the high mass of the squarks leading to final states boosted in the forward direction is exploited to suppress Standard Model (SM) background. The squark mass can then be reconstructed from the final state objects due to energy and momentum conservation. Table 1 shows the total number of observed events in the individual channels, compared to the prediction in the SM from Monte Carlo generators. No significant deviation of data from the SM is observed in all channels and no evidence of squark production is found.

Using the MSSM and the minimal supergravity model (mSUGRA), limits are set using a frequentist approach based on likelihood ratios. The SUSYGEN3 [2] generator is used for the calculation of branching ratios into the analysed squark decay final states as well as the simulation of signal events.

In the MSSM the masses and mixings of gauginos are defined by the three parameters: the ratio of Higgs vacuum expectation values, $\tan \beta$; the Higgs mixing parameter $\mu$; the mass scale $M_{2}$ of $S U(2)$ sparticles. Masses of squarks are free parameters and slepton masses are set to $90 \mathrm{GeV}$. Figure 1 shows the result of scans of scenarios for $70<M_{2}<350 \mathrm{GeV}$ and $-300<\mu<300 \mathrm{GeV}$ at fixed $\tan \beta=6$ for $\lambda_{1 j 1}^{\prime}$ and $\lambda_{11 k}^{\prime}(j, k=1,2,3)$. Shown are the limits excluded at $95 \% \mathrm{CL}$ for all investigated scenarios with a solid line and for the best exclusion achieved in part of the scenarios with a dashed line. Indirect limits from low energy precision observables are shown as well as the limit from the previous $\mathrm{H} 1$ analysis [3]. For Yukawa-type couplings $\lambda_{1 j 1}^{\prime}$ or $\lambda_{11 k}^{\prime}$ of electromagnetic strength $\sqrt{4 \pi \alpha_{\mathrm{em}}}=0.3$, up-type squarks are excluded up to masses of $275 \mathrm{GeV}$ at $95 \% \mathrm{CL}$, with down-type squarks further excluded up to masses of $290 \mathrm{GeV}$.

In mSUGRA all masses and mixings are determined completely by the common mass of scalar sparticles $m_{0}$; the common mass of fermionic sparticles $m_{1 / 2}$; the common trilinear coupling $A_{0}$; the ratio of Higgs vacuum expectation values, $\tan \beta$; the sign of the Higgs mixing parameter $\mu$. Figure 2 (left) shows constraints on the parameters $\left(m_{0}, m_{1 / 2}\right)$ when $R_{p}$ couplings of electromagnetic coupling strength are assumed. The hatched blue region gives the excluded domain for first and second generation squarks and the green region indicates where third generation squarks could be excluded at $95 \%$ CL. Curves of constant squark masses and partly complementary constraints 


\begin{tabular}{|c|c|c|c|c|c|c|c|c|c|c|c|}
\hline \multirow{3}{*}{$\begin{array}{c}\text { Selection } \\
\text { Channel } \\
e q\end{array}$} & \multicolumn{4}{|c|}{$e^{-} p\left(183 \mathrm{pb}^{-1}\right)$} & \multicolumn{4}{|c|}{$e^{+} p\left(255 \mathrm{pb}^{-1}\right)$} & \multirow{2}{*}{\multicolumn{3}{|c|}{$\begin{array}{c}\text { Range of Signal } \\
\text { Efficiencies }\end{array}$}} \\
\hline & \multirow{2}{*}{$\frac{\text { Data }}{3121}$} & \multicolumn{3}{|c|}{ SM Expectation } & \multirow{2}{*}{$\begin{array}{l}\text { Data } \\
2946\end{array}$} & \multicolumn{3}{|c|}{ SM Expectation } & & & \\
\hline & & 3215 & . & 336 & & 2899 & + & 302 & $30 \%$ & - & $40 \%$ \\
\hline$v q$ & 2858 & 2983 & \pm & 358 & - & & - & & $50 \%$ & - & $60 \%$ \\
\hline$e M J(\mathrm{RC})$ & 147 & 158.3 & \pm & 23.9 & 140 & 146.0 & \pm & 21.4 & $10 \%$ & - & $40 \%$ \\
\hline$e M J(\mathrm{WC})$ & 0 & 1.3 & \pm & 0.3 & 1 & 0.6 & \pm & 0.4 & $5 \%$ & - & $20 \%$ \\
\hline$e e M J$ & 0 & 1.5 & \pm & 0.5 & 2 & 1.7 & \pm & 0.5 & $5 \%$ & - & $35 \%$ \\
\hline$e \mu M J$ & 0 & 0.03 & \pm & 0.02 & 0 & 0.03 & \pm & 0.03 & $5 \%$ & - & $15 \%$ \\
\hline$e v M J$ & 3 & 5.6 & \pm & 1.2 & 5 & 8.2 & \pm & 2.0 & $5 \%$ & - & $40 \%$ \\
\hline$v M J$ & 204 & 235.5 & \pm & 63.3 & 113 & 134.0 & \pm & 33.8 & $5 \%$ & - & $15 \%$ \\
\hline$v \mu M J$ & 0 & 0.04 & \pm & 0.02 & 0 & 0.06 & \pm & 0.03 & $5 \%$ & - & $20 \%$ \\
\hline
\end{tabular}

Table 1: Total numbers of selected events, SM expectations and ranges of signal efficiencies for the squark decay channels considered in $e^{-} p$ and in $e^{+} p$ collisions. The range of signal efficiencies gives the extreme values for squark masses ranging from $100 \mathrm{GeV}$ to $290 \mathrm{GeV}$ and gaugino masses ranging from $30 \mathrm{GeV}$ up to the squark mass. The $v q$ channel is not relevant for $e^{+} p$ data since the $\tilde{u}_{L}$-type squarks produced in $e^{+} p$ do not undergo this decay. Only $\tilde{d}_{R}$-type squarks, which are produced dominantly in $e^{-} p$ collisions, can undergo direct decay leading to a $v q$ final state. The total error on the SM prediction is determined by adding the effects of all model and experimental systematic uncertainties in quadrature.

from other colliders are indicated for comparison.

The $\tan \beta$ dependence of mSUGRA exclusion limits is studied assuming a unified common mass $M=m_{0}=m_{1 / 2}$. The region below the lines in figure 2 (right) is excluded at $95 \% \mathrm{CL}$ for couplings comparable to the electromagnetic coupling strength for each of the indicated flavours. Results for the first two generations of up-type and down-type squarks are independent of $\tan \beta$, while mixing effects with increasing $\tan \beta$ lead to masses for stop quarks lower than for first and second generation up-type squarks.

In summary, the existence of $\tilde{u}_{L}$-type and $\tilde{d}_{R}$-type squarks of all three generations with masses up to $275 \mathrm{GeV}$ and $290 \mathrm{GeV}$, respectively, is excluded at the 95\% CL for Yukawa couplings of electromagnetic strength. These mass limits set the most stringent direct bounds on $\lambda_{1 j 1}^{\prime}$ and $\lambda_{11 k}^{\prime}$. For lower squark masses, the results improve the indirect bounds set by low-energy experiments. Exclusion limits are also derived in the mSUGRA model, and are competitive with and complementary to those derived at the LEP and Tevatron colliders.

\section{References}

[1] F. D. Aaron et al., Eur. Phys. J. C 71 (2011) 1572 [arXiv:1011.6359 [hep-ex]].

[2] S. Katsanevas and P. Morawitz, Comput. Phys. Commun. 112 (1998) 227 [hep-ph/9711417]; N. Ghodbane, S. Katsanevas, P. Morawitz and E. Perez, SUSYGEN 3, hep-ph/9909499.

[3] A. Aktas et al. [H1 Collaboration], Eur. Phys. J. C36 (2004) 425 [hep-ex/0403027].

[4] R. N. Mohapatra, Phys. Rev. D34 (1986) 3457;

J. D. Vergados, Phys. Lett. B184 (1987) 55;

M. Hirsch, H. V. Klapdor-Kleingrothaus and S. G. Kovalenko, Phys. Lett. B352 (1995) 1

[hep-ph/9502315]; 
M. Hirsch, H. V. Klapdor-Kleingrothaus and S. G. Kovalenko, Phys. Rev. Lett. 75 (1995) 17; M. Hirsch, H. V. Klapdor-Kleingrothaus and S. G. Kovalenko, Phys. Rev. D53 (1996) 1329 [hep-ph/9502385].

[5] R. Barbier et al., Phys. Rept. 420 (2005) 1.

[6] V. Barger, G. F. Giudice and T. Han, Phys. Rev. D40 (1989) 2987.

[7] P. Langacker, Phys. Lett. B256 (1991) 277.

[8] M. Acciarri et al. [L3 Collaboration], Eur. Phys. J. C19 (2001) 397 [hep-ex/0011087].

[9] B. Abbott et al. [D0 Collaboration], Phys. Rev. Lett. 83 (1999) 4476 [hep-ex/9907019]. 

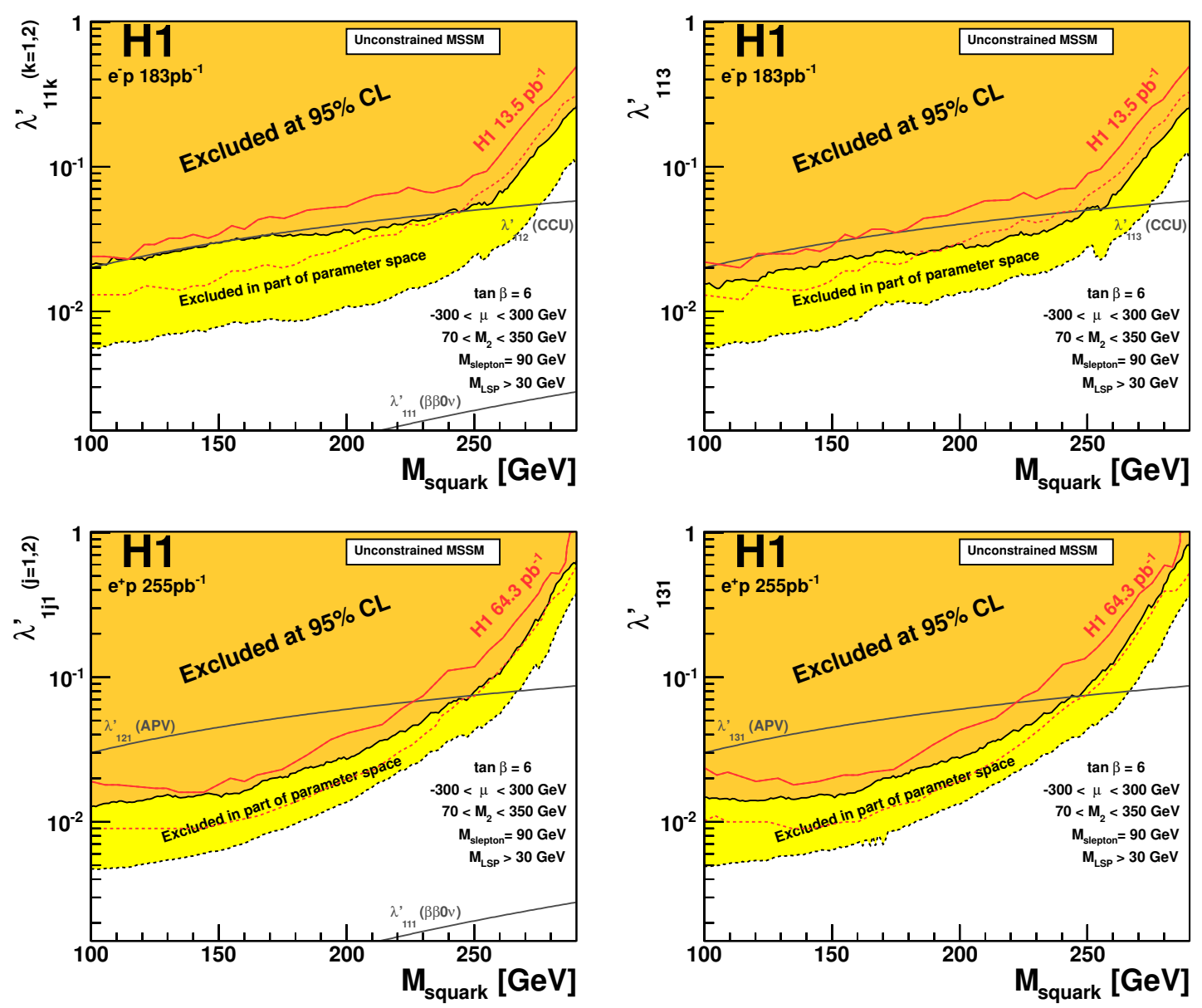

Figure 1: Exclusion limits (95\% CL) on $\lambda_{11 k}^{\prime}$ (top row) and $\lambda_{1 j 1}^{\prime}$ (bottom row) for $k=1,2$ (left) and $k=3$ (right) as a function of the squark mass from a scan of the MSSM parameter space. The dark filled region indicates values of the coupling $\lambda_{11 k}^{\prime}$ excluded in all investigated scenarios whereas the light filled region is excluded only in part of the scenarios. Indirect limits from neutrinoless double beta decay experiments $(\beta \beta 0 v)[4,5]$, tests of charged current universality (CCU) $[6,5]$ and atomic parity violation (APV) $[7,5]$ are also shown. For comparison, the corresponding limits from the previous H1 analysis [3] are also indicated in all scans. 

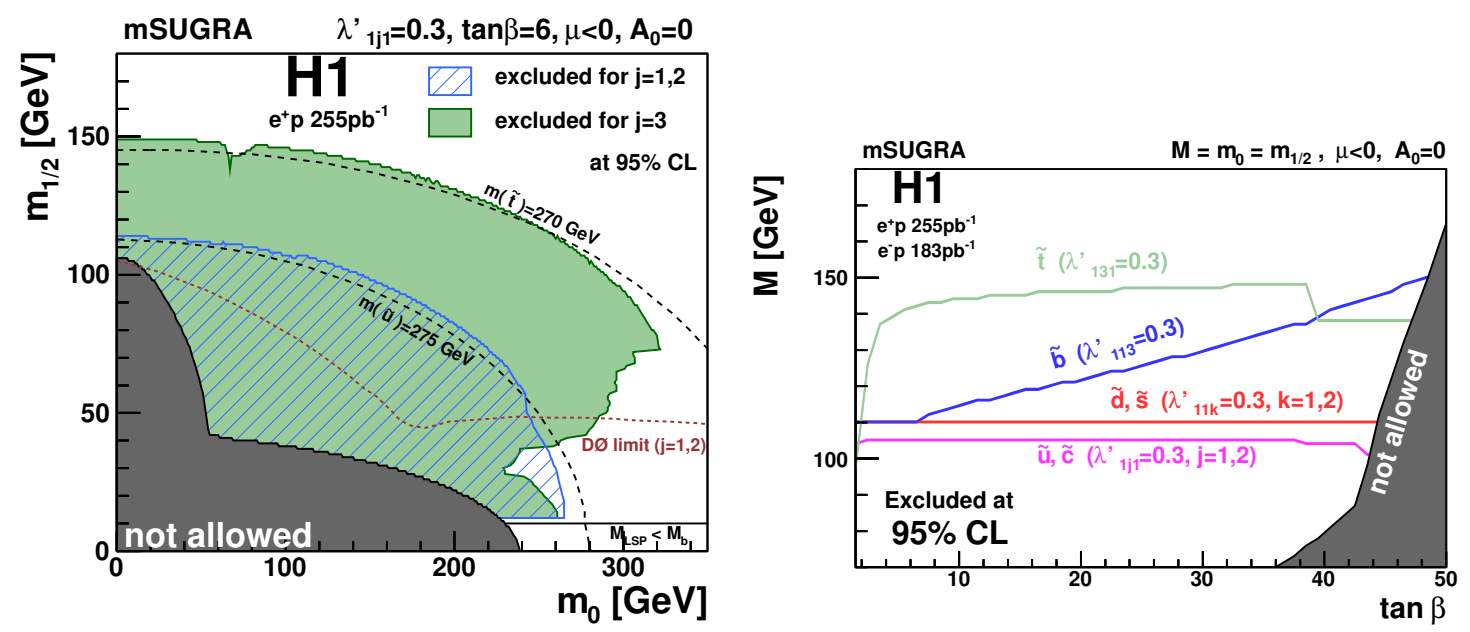

Figure 2: Left: Exclusion limits (95\% CL) in the $m_{0}, m_{1 / 2}$ plane assuming $\lambda_{1 j 1}^{\prime}=0.3$ for $\tan \beta=6$ for $j=1,2$ (hatched region) and $j=3$ (light filled region). Curves of constant squark mass are illustrated for $m(\tilde{u})=275 \mathrm{GeV}$ and $m(\tilde{t})=270 \mathrm{GeV}$. Also indicated are constraints obtained by the $\mathrm{L} 3$ experiment at LEP [8] and the DØ experiment at the Tevatron [9]. The dark filled region labelled as "not allowed" indicates where no REWSB solution is possible or where the LSP is a sfermion. Right: Exclusion limits for $M=m_{0}=m_{1 / 2}$ in mSUGRA as function of $\tan \beta$. Shown are the $95 \%$ CL exclusion domains for the model parameters from the production of squarks of first and second generation $(\tilde{u}, \tilde{c}$ and $\tilde{d}, \tilde{s})$ and of third generation $(\tilde{t}, \tilde{b})$ assuming a value of $\lambda^{\prime}=0.3$ for the respective coupling. The area below the curves is excluded. The dark filled region region labelled as "not allowed" indicates where no REWSB solution is possible or where the LSP is a sfermion. 